\title{
A comparative morphological study of the spinous processes of the sixth and seventh cervical vertebrae in extinct and extant species of Equus
}

\author{
Sharon May-Davis*, Robert Hunter, and Wendy Y. Brown \\ University of New England, Canine and Equine Research Group, Armidale, New South Wales, Australia \\ maydavis@bigpond.com; anthropal@outlook.com; wbrown@une.edu.au
}

\begin{abstract}
The lateral profile of the 6th and 7th cervical spinous processes (CSPs) were examined in four extant species of Equus $(\mathrm{n}=33)$; E. caballus $(\mathrm{n}=26)$, E. przewalskii $(\mathrm{n}=3)$, E. quagga boehmi $(\mathrm{n}=1)$, E. asinus $(\mathrm{n}=3)$ and compared to pre-domesticated Equus specimens $(\mathrm{n}=66)$ representing three known species; E. occidentalis $(\mathrm{n}=56)$, E. mosbachensis $(\mathrm{n}=2)$, E. curvedins/insulatis $(\mathrm{n}=1)$ and unknown Equus species $(\mathrm{n}=7)$ from five museums. Six common morphological profiles were revealed: cuneate, curvate, falcate, rudimentary, scalenate, and truncate. For the 6th CSP, the distribution of these morphologies amongst extant Equus is: cuneate, only E. asinus; curvate, E. caballus and E. przewalskii always in combination with ligamentum lamina nuchae (lig. lamina nuchae) attachments from the 2nd through to 7th CSP inclusive; falcate, E. caballus and E. przewalskii always in combination with lig. lamina nuchae attachments from the 2nd through to 7 th CSP inclusive; rudimentary, E. caballus always associated with lig. lamina nuchae attachments from the 2nd through to 5th CSP inclusive; scalenate, E. caballus in association with lig. lamina nuchae attachments from the 2 nd through to 5th $(\mathrm{n}=11)$ or 7 th $(\mathrm{n}=4)$ CSP inclusive; truncate, not present. The 6th CSP in museum specimens of Equus exhibits one of four profiles: cuneate $(n=10)$, curvate $(n=14)$, scalenate $(n=11)$ and truncate $(n=4)$. For the 7 th CSP, the distribution of these morphologies amongst extant Equus is: curvate, E. caballus mostly associated with lig. lamina nuchae attachments from the 2 nd through to 5th CSP inclusive, falcate, E. caballus mostly associated with lig. lamina nuchae attachments from the 2nd through to 7th CSP inclusive, scalenate, E. caballus associated with lig. lamina nuchae attachments from the 2nd through to 5th CSP inclusive. Only Rancho La Brea and Tar Pits Museum provided samples suitable for examination of the 7th CSP. These exhibited four profiles: curvate $(n=7)$, falcate $(n=11)$, scalenate $(n=2)$, and truncate $(n=7)$. These findings suggest that the lateral profile of the 6th CSP is of potential use in identification of species; attachments of the lig. lamina nuchae alter the morphology of the 6th and 7th CSP; and that attachments of the lig. lamina nuchae on the 2nd to 7th CSP were likely present in species of Equus prior to domestication.
\end{abstract}

\section{INTRODUCTION}

The number of cervical vertebrae in mammals became fixed more than 200 million years ago (mya); the canonical number of seven cervicals is well established by palaeontological evidence (Buchholtz et al. 2012; Arnold 2020). The mammalian cervical spine, considered as a morphologically static feature (Arnold et al. 2017), typically includes dorsally positioned spinous processes on some or all of the 2nd to 7th cervical vertebrae (Zsoldos and Licka 2015; Arnold 2020). The North American Eocene perissodactyl
Hyracotherium (Buchholtz et al. 2012; MacFadden 1992) bears blade-like spinous processes on the 4th to 7 th cervical vertebrae (Wood et al. 2011) (Fig. 1A). This morphological trait is still retained in two of three extant perissodactyl families, namely Tapiridae and Rhinoceridae (Fig. 1C, D). Members of the third family, Equidae (the hippomorphs) lack distinct spinous processes on the 3 rd to 5 th, with those on the 6th and 7th greatly reduced (Getty 1975) (Fig. 1B). Cervical spinous processes (CSPs) provide important attachment sites for neck and back musculature, as well as ligamentous structures such as the lig. lamina nuchae 

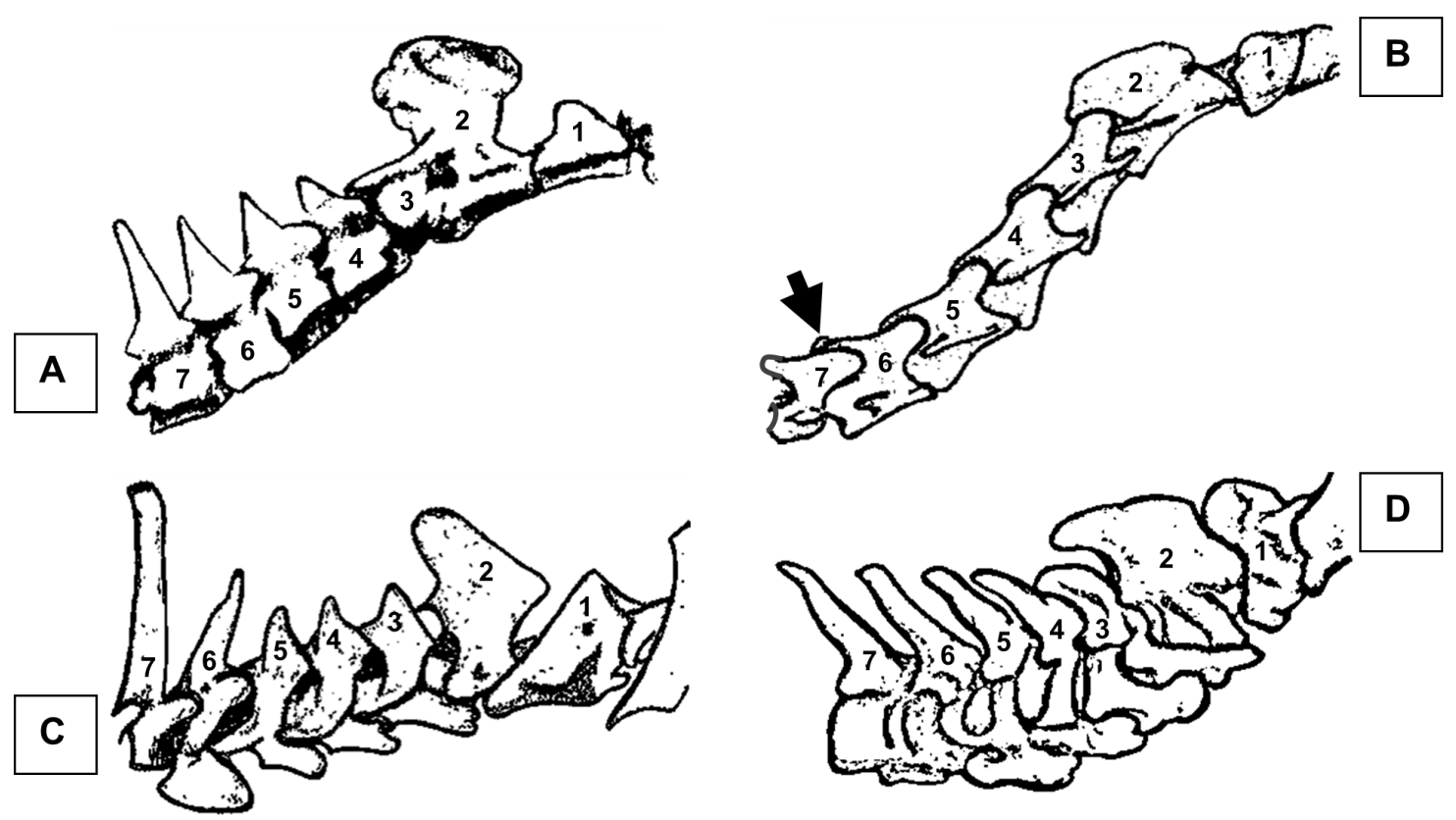

Figure 1. Cervical spinous processes of perissodactyls; A, Eocene Hyracotherium; B, modern domestic horse - Equus caballus breed Thoroughbred (7th arrowed) - note the normal morphological presentation of the 7 th spinous process and its position between the articulation of the 6th and 7th cervical vertebrae; C, Malayan tapir - Tapirus indicus; D, Sumatran rhinoceros Dicerorhinus sumatrensis. (Sketches by 1st author).

(Getty 1975; Wood 2011; Zsoldos and Licka 2015; Arnold 2020). This ligament attaches to the CSPs from the 2 nd to 7th in extant Tapiridae and according to early pre 1900s anatomic literature also in E. caballus in Equidae (Muir 1871; Wood et al. 2011; Singh 2018; May-Davis et al. 2018; Arnold 2020). A similar morphology has been reconstructed for the Eocene perissodactyl Hyracotherium (Wood et al. 2011). Eocene perissodactyls were browsers, and the morphology of the cervical vertebrae in Hyracotherium reflects the orientation and magnitude of forces applied to its cervical ' $S$ ' shaped spine (MacFadden 1992; Wood et al. 2011; Zsoldos and Licka 2015, Arnold 2020). This orientation of the spinous processes allows for greater dorsoventral flexion, an ideal characteristic for browsing (Wood et al. 2011). By the mid-Miocene it is believed that Merychippus, a hippomorph descendant of Hyracotherium, had evolved a primarily grazing habit (MacFadden, 1992) and consequently the forces acting upon the cervical column were significantly altered (Nussey et al. 2007; Zsoldos and Licka 2015). This is evidenced by the significant reduction in the spinous processes from those of Hyracotherium to Merychippus with only the 6th and 7th still presenting a raised apex/eminence in the latter (personal observation of museum specimens and replicas) (Fig. 2).

A reduction in lig. lamina nuchae attachments from the 6th and 7th CSP has been reported in 30 breeds of domestic E. caballus; however, this derived morphology does not extend to all breeds of E. caballus or other extant species of Equus (May-Davis and Kleine 2014, May-Davis et al.
2018, 2020b, c). In addition, where the attachments of the lig. lamina nuchae extend from the 2nd to 7 th CSP in extant species of Equus, the lateral profiles of the 6th and 7th CSP differ from those in extant species of Equus where attachments of the ligament are restricted to the 2 nd to 5 th CSP (May-Davis et al. 2018; 2020a). Although the evolutionary development of the equine limb has been studied extensively, an equivalent treatment of the cervical spine is still lacking (Zsoldos and Licka 2015).

In concurrence with Zsoldos and Licka (2015), this study investigates the lateral morphological profile of the 6th and 7th CSPs in four extant species of Equus. After establishing the common profiles, we compare these species with museum specimens of pre-domesticated Equus. The aim is to: (i) identify potential species-specific traits to assist palaeontologists in the identification of museum specimens in Equus and (ii) investigate evidence for the presence or absence of attachments of the lig. lamina nuchae from the 2nd to 7th CSP in pre-domesticated Equus.

\section{METHODS}

Preparation of Extant Specimens Equus caballus, Equus przewalskii, Equus quagga boehmi and Equus asinus

Ethical Statement: No equids were euthanised for the purpose of this study and all observations were obtained postmortem. 

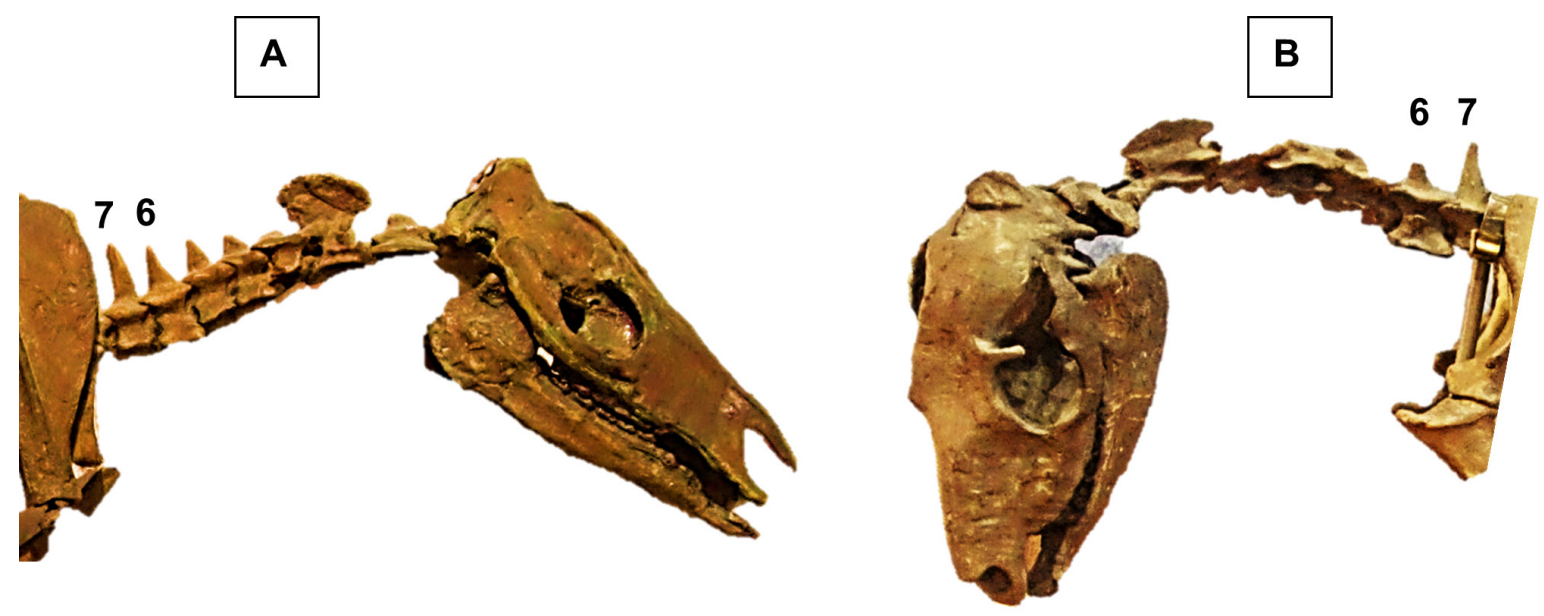

Figure 2. Cervical vertebrae in two extinct equids noting the 6th and 7th spinous processes; A, Eocene Hyracotherium; B, Miocene Merychippus. (Photographs by 1st author).

Anatomical Terminology: The anatomical terms for this manuscript followed the terminology from Nomina Anatomica Veterinaria 6th edition (Gasse et al. 2017), except regarding tuberculum dorsale. Here the authors revert to anatomical terminology from Getty (1975) citing the equivalent skeletal structure as spinous process. Descriptive terminology of the spinous process notes the dorsal tip as 'eminence' when rounded, and 'apex' when pointed.

Functional Anatomy: The lig. lamina nuchae consists of a bilateral sheet of yellow elastin ligamentous fibres that arise from the spinous processes of the 2 nd and 3 rd thoracic vertebrae and the ligamentum funiculus nuchae, after which they traverse cranioventrally and insert on the CSPs from the 2 nd to 7 th cervicals in E. przewalskii, E. quagga boehmi and E. asinus. In modern (domestic) E. caballus, the lig. lamina nuchae primarily inserts from the 2 nd to 5 th CSPs, with rare variations extending to the 6th, and/or 7th CSP. Although reports of primitive and isolated breeds of E. caballus and close descendants of Equus ferus ferus often present the lig. lamina nuchae attachments from the 2nd to 7th CSPs (May-Davis et al. 2018, 2020b, c).

As the bilateral sheets of lig. lamina nuchae descend towards the CSPs, they form digitations prior to attachment; the only exception is E. asinus in which the lig. lamina nuchae remains an unbifurcated sheet (Sisson 1975; MayDavis and Kleine 2014; May-Davis et al. 2018, 2020a). The function of the lig. lamina nuchae is to support and stabilize the cervical vertebrae while assisting locomotive gaits (Gellman and Bertram 2002; Dunbar et al. 2008). In the four extant species, the 6th and 7th CSPs are dorsal projections on the raised, sagittal ridge on the dorsal surface of the vertebral lamina. They present with a cranial and caudal margin that is demarcated by an apex or eminence. The height and orientation of the CSPs are individually variable in Equus, though Getty (1975) describes the 6th CSP in E. caballus as a rudimentary profile, while the 7 th is raised. Cervical spinous processes function to provide attachment sites and anchorage for musculature and ligamentous structures to act on during locomotion, stance, and head relocation for the vestibular apparatus (Getty 1975; Sisson 1975a, b; Haussler 2016).

Animal Details: A total of 33 representative specimens of four species of equids were dissected and examined: $E$. caballus ( $\mathrm{n}=26)$, E. przewalskii $(\mathrm{n}=3)$, E. quagga boehmi $(\mathrm{n}=1)$ and $E$. asinus $(\mathrm{n}=3)$. Equus caballus specimens are divided into two categories that are determined by the number of CSPs to which the lig. lamina nuchae attaches, either from the 2 nd to 5 th CSPs $(n=17)$ or 2 nd to 7 th CSPs $(n=9)$. The category 2 nd to 5 th comprises the following breeds: Crossbred $(\mathrm{n}=1)$; Dutch Konik $(\mathrm{n}=2)$; Exmoor ( $\mathrm{n}=2)$; Quarter horse $(\mathrm{n}=2)$; Shetland $(\mathrm{n}=1)$; Thoroughbred $(n=6)$; Warmblood $(n=2)$; Welsh Mountain Pony $(\mathrm{n}=1)$. The category 2 nd to 7 th comprises isolated breeds: Australian Stock Horse $(\mathrm{n}=2)$; Bosnian Mountain Horse $(\mathrm{n}=1)$; Dutch Konik $(\mathrm{n}=2)$; Waler $(\mathrm{n}=1)$; Yonaguni pony $(\mathrm{n}=3)$. It should be noted that $E$. przewalskii, $E$. quagga boehmi and $E$. asinus present with lig. lamina nuchae attachments from the 2 nd to 7 th CSPs. The ages range from $16 \mathrm{mths}-23$ years and comprised of 16 males and 18 females.

Source: Equua caballus were obtained from either an abattoir, or postmortem following death by natural causes or veterinary recommendation for euthanasia in Australia, Japan, Slovenia, Netherlands, and United Kingdom. Equus asinus was obtained from an abattoir in Australia; E. przewalskii from Hortobagy Nature Reserve in Hungary and $E$. quagga boehmi from Emmen Zoo in the Netherlands. 
May-Davis et al. - Comparative morphology of spinous processes in Equus

Dissections: Protocols established by May-Davis et al. (2018) resect the neck musculature to reveal the attachments of the lig. lamina nuchae to the CSP. Further resection of the lig. lamina nuchae reveals the profiles of the 6th and 7th CSPs.

Maceration, Degreasing and Photography: Twentyfour specimens required maceration postmortem following the protocols established by May-Davis et. al (2020a), observations of the remaining nine specimens were in situ. The photographic view is left lateral for continuity and individual specimens follow those protocols established by May-Davis et. al (2020a).

\section{Fossil Specimens}

Museums: Five museums with suitable specimens provided access for the study. Only those 6th and 7th cervical specimens with complete CSP or morphology sufficiently intact were examined. In total 66 specimens (6th $\mathrm{n}=39$; 7 th $n=27$ ) are included. Specimens of the 6th cervical vertebrae were obtained from: Natural History Museum Berlin (unidentified Equus species, n=6); Rancho La Brea Tar Pits and Museum Los Angeles (E. occidentalis, $\mathrm{n}=29$ ); Monrepos Archeological Research Center and Museum for Human Behavioral Evolution Germany (E. mosbachensis, $\mathrm{n}=2$ ); Natural History Museum Rotterdam (unidentified Equus species, $\mathrm{n}=1$ ) and the Swedish Royal Museum and Natural History Stockholm (E. curvedins/ insulatis, $\mathrm{n}=1$ ). Additionally, suitable specimens of the 7th cervical vertebra were examined at Rancho La Brea Tar Pits and Museum Los Angeles (E. occidentalis $\mathrm{n}=27$ ).

Photography: In total, 66 specimens were photographed following the protocol established by May-Davis et. al (2020a), except specimens were handheld. Photographic images are in left lateral view only for continuity.

\section{Describing the Left Lateral Profile of the 6th and 7th Cervical Spinous Processes}

This description of the lateral profiles of the 6th and 7th cervical spinous processes in four species of extant Equus and museum specimens of pre-domesticated Equus, uses the basic terminology applied by Meyer et al. (2015) in a comparative study of thoracic vertebrae in hominids. The cited authors compared the laminar of the thoracic vertebra of Australopithecus afarensis (specimen AL 288-1 am) to that of Papio and noted a morphological similarity exemplified by a triangular shape, referring to it as the superior laminar trigon.

Here we follow similar protocols where the profiles of the spinous processes are characterised according to the shape bounded by four basic morphological features: baseline of the spinous process, cranial margin, eminence/apex and caudal margin (Fig. 3).

The baseline for each profile is determined with a straight line extending from the apex of the vertebral arch to where the caudal aspect of the dorsal spine diminishes and blends into either the sagittal ridge or the caudal border of the vertebra (whichever comes first). The cranial margin extends from the eminence/apex to the cranial aspect of the baseline. The caudal margin extends from the eminence/ apex to the caudal aspect of the baseline.

The outline for each margin creates a shape, either convex, concave or straight. The combination of the four morphological features and their outlines (baseline, cranial and caudal margin, and eminence/apex) provides a shape that can be related to either a biological or botanical profile (Arnold, 2021; Pellow, Henwood and Carolin, 2010).

\section{RESULTS}

\section{Spinous process morphology of the 6th and 7 th cervical vertebrae}

Six primary lateral profiles were identified as representational of the 6th and/or 7th CSP. Each profile was indicative of a botanical or biological shape: cuneate (wedge shaped), curvate (a curved form), falcate (sickle or hooked shaped), rudimentary (underdeveloped or no profile), scalenate (unequal sides), and truncate (cut off across the top) (Tab. 1). The specific profiles observed were dependent on the number of CSPs to which the lig. lamina nuchae attaches. Two distinct attachment patterns were

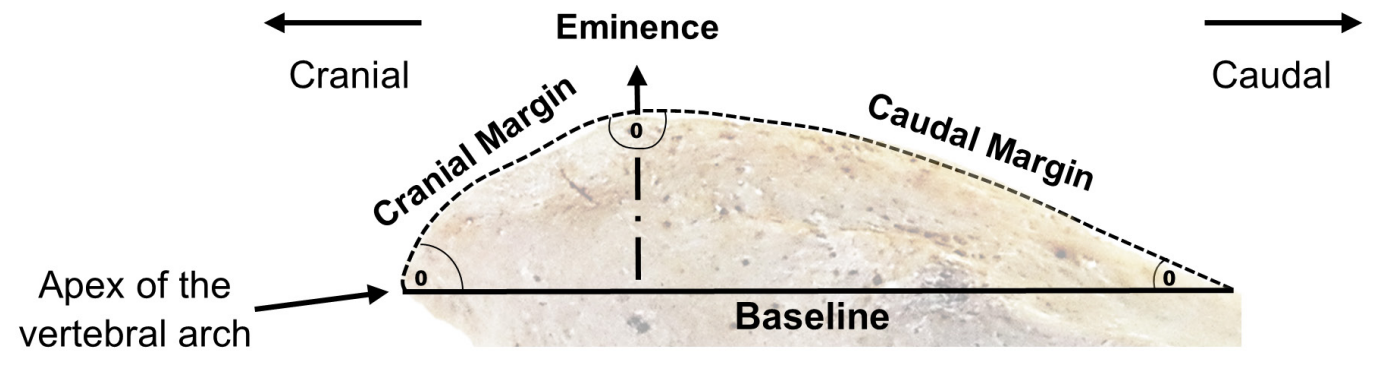

Figure 3. Cervical spinous process presenting four morphological features: baseline of the spinous process, cranial margin, eminence, and caudal margin. 
Table 1. Summary of six primary profiles observed in the 6 th, and/or 7 th cervical spinous process of four extant species of Equus and museum specimens of extinct Equus.

\section{Cuneate}

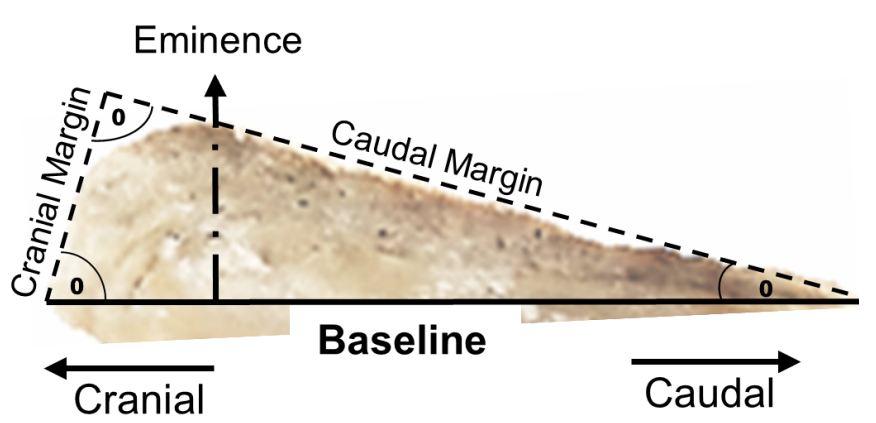

Equus asinus - C6 specimen 15 years old

Profile height: High to baseline

Cranial ${ }^{0}:>60$

Eminence ${ }^{0}$ : Intersection of the lines associative to the cranial and caudal margins 90 degrees

Caudal ${ }^{0}:<30$

Cranial Margin: Lesser than caudal in length

Eminence description: Rounded or apex

Caudal Margin: Greater than cranial in length

\section{Curvate}

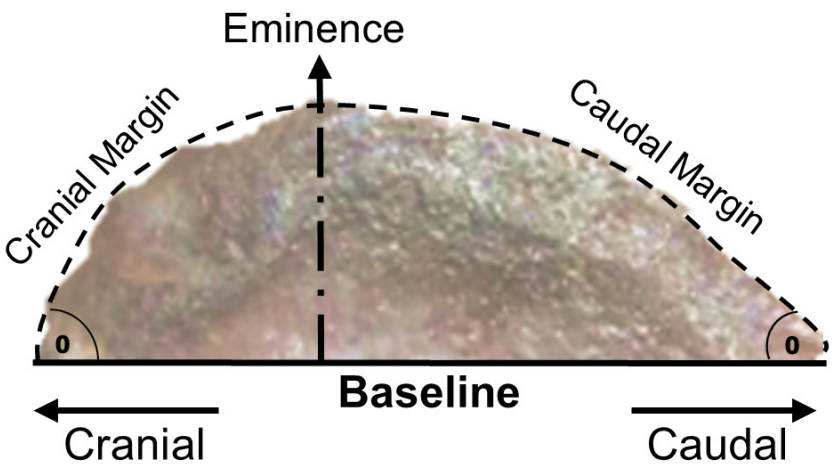

Equus occidentalis - C6 specimen Rancho La Brea and Tar Pits specimen No. 4 D2-99

Profile height: High to baseline

Cranial ${ }^{0}$ : N/A

Eminence ${ }^{0}: \mathrm{N} / \mathrm{A}$

Caudal ${ }^{0}$ : N/A

Cranial Margin: Convex to the eminent line

Eminence description: Rounded

Caudal Margin: Convex to the eminent line

Falcate

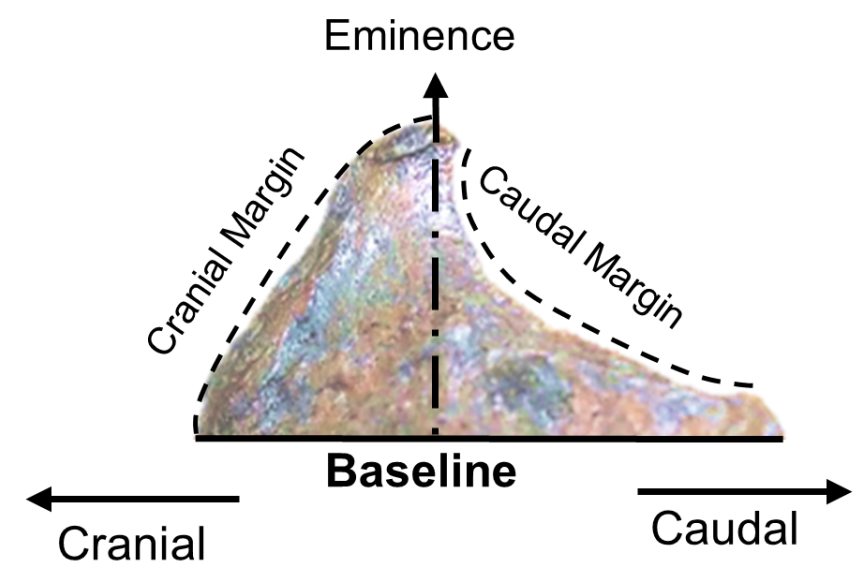

Equus occidentalis - C7 specimen Rancho La Brea and Tar Pits Specimen No. 3 C3-4, 6.5

Profile height: High to baseline

Cranial ${ }^{0}: \mathrm{N} / \mathrm{A}$

Eminence ${ }^{0}: \mathrm{N} / \mathrm{A}$

Caudal ${ }^{0}$ : N/A

Eminence description: Rounded or apex

Cranial Margin: Predominantly convex to the eminent line

Caudal Margin: Predominantly concave to the eminent line

\section{Rudimentary}

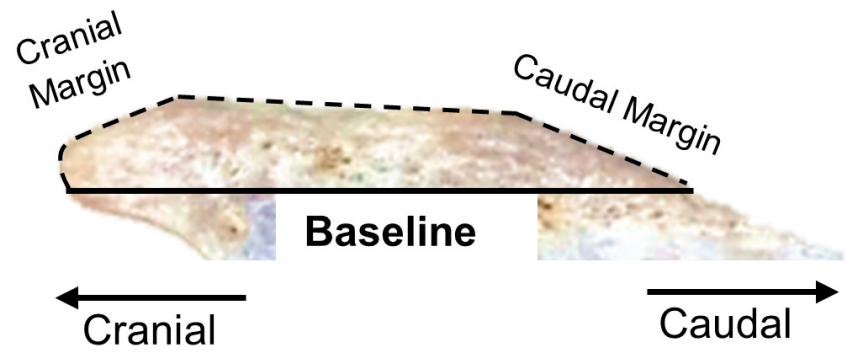

Equus caballus - C6 specimen - Shetland pony $>6$ years old Profile height: Low to baseline

Cranial ${ }^{0}: \mathrm{N} / \mathrm{A}$

Eminence ${ }^{0}: \mathrm{N} / \mathrm{A}$

Caudal ${ }^{0}$ : N/A

Cranial Margin: Lesser than caudal in length

Eminence description: Parallel to the baseline - long extended

Caudal Margin: Greater than cranial in length 


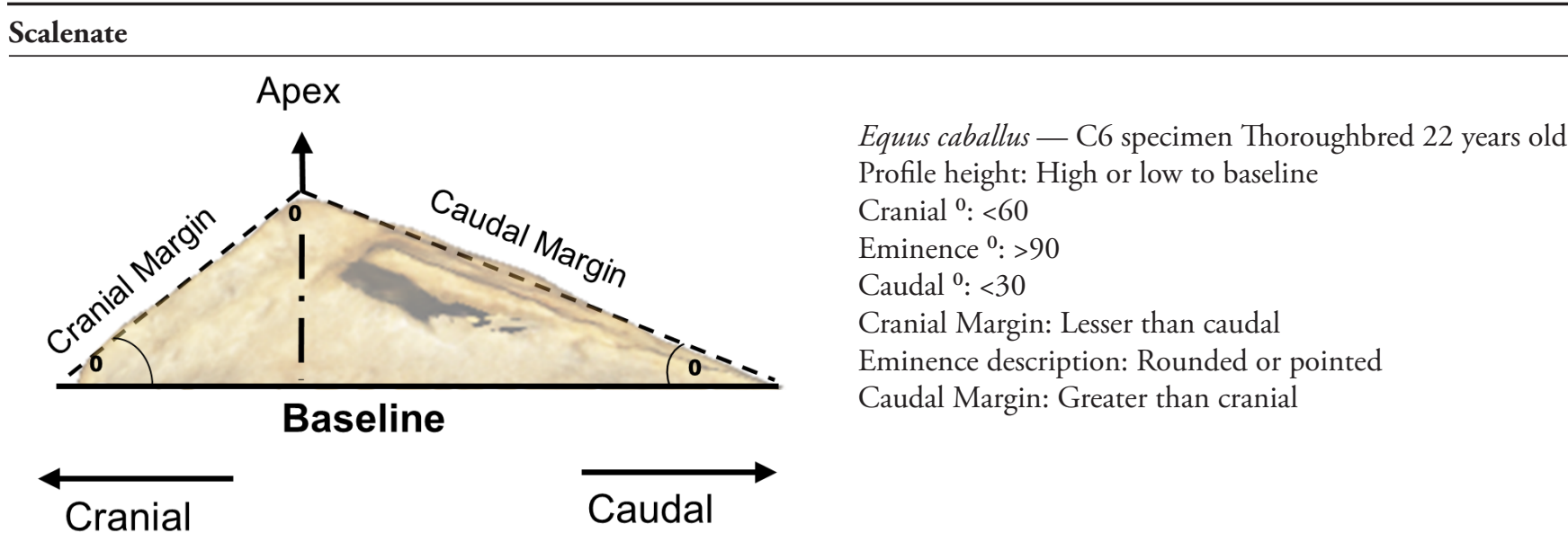

\section{Eminence}

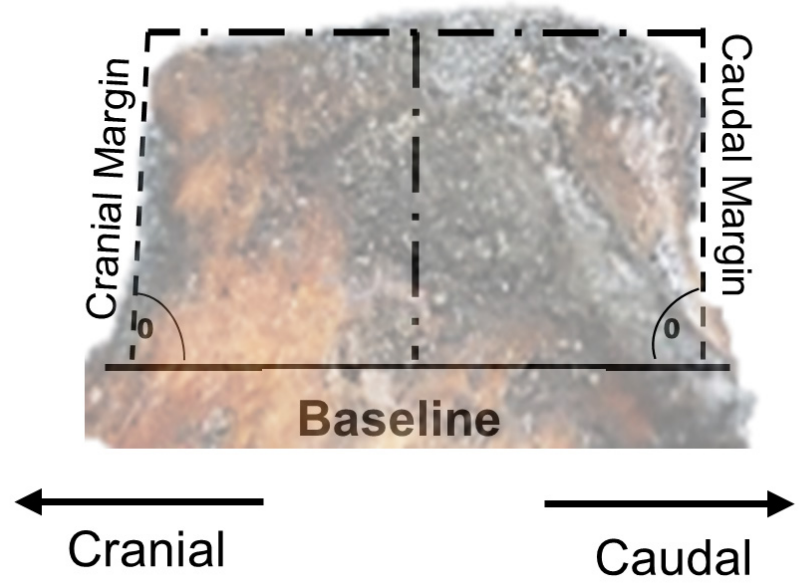

Equus occidentalis - C6 specimen Rancho La Brea and Tar Pits Specimen No. 2-3, F-4, 12

Profile height: Truncate

Cranial ${ }^{0}$ : Can be $<90$

Eminence ${ }^{0}: \mathrm{N} / \mathrm{A}$

Caudal ${ }^{0}$ : Can be $>90$

Cranial Margin: Relatively straight and parallel to the caudal margin.

Eminence description: Relatively straight and can be parallel to the baseline or might present a cranial tilt.

Caudal Margin: Relatively straight and parallel to the cranial margin. observed: the lig. lamina nuchae attachments from the 2nd to 5th CSPs, or from the 2nd to 7th CSPs (Tab. 2). Small variations in profile shape were observed yet they remained within the described guidelines (Tab. 1). Some profiles were further noted as high (where the CSP forms a distinct profile on the dorsal surface of the neural arch) and low (where the height of the profile is close vertically to the sagittal midline).

\section{The 6th cervical spinous process of four extant} species and museum specimens of Equus: E. callabus exhibits two distinct attachment patterns of the lig. lamina nuchae, 2nd to 5th, and 2nd to 7th. The 6th CSP of $E$. caballus (with lig. lamina nuchae attachments from the 2nd to 5 th or 7 th cervical vertebrae), E. asinus (Ea), E. przewalskii (Ep), E. quagga boehmi (Eqb) and museum specimens present all six primary profiles (Fig. 4).

E. callabus (2nd to 5th): The 6th CSP present two low morphological profiles along the sagittal midline - rudimentary 10/17 (59\%) and scalenate 7/17 (41\%). Incomplete non-functional lig. lamina nuchae were present in 10 specimens 10/17 (59\%).

E. callabus (2nd to 7th): The 6th CSP present two high morphological profiles along the sagittal midline - scalenate 4/10 (40\%) and curvate 6/10 (60\%). Both profiles are more robust in this category than in category 2nd to 5th, especially the eminence/apex of the scalenate profile, where the lig. lamina nuchae attachments occur at one location rather than those with curvate morphology.

Equus asinus: The 6th CSP present one morphological profile that spans the entire length of the sagittal midline - cuneate $3 / 3$ (100\%). The lig. lamina nuchae (which attaches to the 2nd to 7 th CSP) attaches to the cranial margin, eminence and caudal margin of the spinous process that spanned the entire length of the cervical vertebra from the cranial to caudal border. The cuneate profile appears morphologically robust. 
Table 2. Summary of six primary profiles observed in the 6th, and/or 7 th cervical spinous in association with two different ligamentum lamina nuchae attachment patterns (2nd to 5 th and 2 nd to 7 th).

Breed Lig. lamina nuchae Cuneate Curvate Falcate Rudimentary Scalenate Truncate

Australian Stock Horse

Australian Stock Horse

$2-7$

Bosnian Mountain Horse

Crossbred

Dutch Konik

Dutch Konik

Dutch Konik

Dutch Konik

Exmoor

Exmoor

Quarter Horse

Quarter Horse

Shetland

Thoroughbred

Thoroughbred

Thoroughbred

Thoroughbred

Thoroughbred

Thoroughbred

Waler

Warmblood

Warmblood

Welsh Mountain Pony

Yonaguni Pony

Yonaguni Pony

Yonaguni Pony

Equus asinus

Equus asinus

Equus asinus

Equus przewalskii

Equus przewalskii

Equus przewalskii

Equus quagga boehmi

Australian Stock Horse

Australian Stock Horse

Bosnian Mountain Horse

Crossbred

Dutch Konik

Dutch Konik

Dutch Konik

Dutch Konik

Exmoor

Exmoor

Quarter Horse

Quarter Horse

Shetland

2-7

$2-7$

2-5

2-5

2-5

$2-7$

2-7

2-5

2-5

2-5

2-5

2-5

2-5

2-5

2-5

2-5

2-5

2-5

2-7

2-5

2-5

2-5

2-7

2-7

2-7

2-7

2-7

2-7

2-7

2-7

2-7

2-7

\section{Equus caballus 6th Cervical Vertebra}

Extant Equus 6th Cervical Vertebra

Equus caballus 7th Cervical Vertebra
*

*

$*$

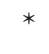

$*$

* (Lig Int)

$*$

$*$

* (Lig Int) 
May-Davis et al. - Comparative morphology of spinous processes in Equus

Table 2 continued.

\begin{tabular}{|c|c|c|c|c|c|c|c|}
\hline Breed & Lig. lamina nuchae & Cuneate & Curvate & Falcate & Rudimentary & Scalenate & Truncate \\
\hline Thoroughbred & $2-5$ & & & & & * & \\
\hline Thoroughbred & $2-5$ & & & & & * & \\
\hline Thoroughbred & $2-5$ & & * & & & & \\
\hline Thoroughbred & $2-5$ & & * & & & & \\
\hline Thoroughbred & $2-5$ & & $*$ & & & & \\
\hline Thoroughbred & $2-5$ & & $*$ & & & & \\
\hline Waler & $2-7$ & & & $*$ & & & \\
\hline Warmblood & $2-5$ & & & & & $*$ & \\
\hline Warmblood & $2-5$ & & * & & & & \\
\hline Welsh Mountain Pony & $2-5$ & & * & & & & \\
\hline Yonaguni Pony & $2-7$ & & & * & & & \\
\hline Yonaguni Pony & $2-7$ & & & $*$ & & & \\
\hline Yonaguni Pony & $2-7$ & & & $*$ & & & \\
\hline
\end{tabular}

Extant Equus 7th Cervical Vertebra

$\begin{array}{ll}\text { Equus asinus } & 2-7 \\ \text { Equus asinus } & 2-7 \\ \text { Equus asinus } & 2-7 \\ \text { Equus przewalskii } & 2-7 \\ \text { Equus przewalskii } & 2-7 \\ \text { Equus przewalskii } & 2-7 \\ \text { Equus quagga boehmi } & 2-7\end{array}$

Lig Int = Very large Ligamenta interspinalia attachment to the caudal margin of the C7 spinous process.

Equus przewalskii: The 6th CSP present two morphological profiles along the sagittal midline - curvate $1 / 3(33 \%)$ and falcate $2 / 3(67 \%)$, with the curvate profile associated only with the youngest animal aged three. The attachments of the lig. lamina nuchae present similar morphology to that of E. quagga boehmi, with little variation and CSP profiles present with a very robust morphology.

Equus quagga boehmi: The very robust 6th CSP presents one morphological profile along the sagittal midline - falcate 1/1 (100\%), which corresponded directly with the cord-like attachment of the lig. lamina nuchae (which attaches from the 2nd to 7 th CSP).

Museum Specimens: The 6th CSP present four morphological profiles along the sagittal midline - cuneate $10 / 39(26 \%)$, curvate $14 / 39(36 \%)$, scalenate (high) $11 / 39(28 \%)$ and truncate $4 / 39(10 \%)$. All CSP profiles present a robust morphology.

\section{The 7th cervical spinous process of four extant} species and museum specimens of Equus: The morphological profile of the 7th CSP of E. caballus (category 2nd to 5 th and 2 nd to 7 th), E. asinus, E. przewalskii, E. quagga boehmi and museum specimens are shown in Figure 5.

E. callabus (2nd to 5th): The 7th CSP present three low morphological profiles along the sagittal midline - curvate 10/17 (59\%), falcate $2 * / 17$ (12\%), and scalenate 5/17
(29\%). The two examples of falcate profiles $\left(^{*}\right)$ are reported in a 22-year-old Thoroughbred female and 15-year-old Quarter Horse male. Both present enlarged ligamenta interspinalia attaching to the entire length of the caudal margin from the eminence to the caudal border of the vertebra with a wide caudal orientation attaching to the entire length of the cranial margin of the 1 st thoracic spinous process.

E. callabus (2nd to 7th): The 7th CSP present two high morphological profiles along the sagittal midline curvate $1 / 10(10 \%)$, and falcate $1 / 9(90 \%)$. The three profiles are more robust in this category than 2 nd to 5 th.

Equus asinus: The lateral profile of the 7th CSP is, in all cases (3/3), falcate. In all cases, the profile is very robust. The attachments of the lig. lamina nuchae span the entire length of the cranial margin, eminence, and caudal margin of the spinous process.

Equus przewalskii: In all cases (3/3), the 7th CSP exhibits a robust, falcate morphology. The lig. lamina nuchae attachments present similar morphology (with lig. lamina nuchae fibres combining to form cord like structures) with little variation between the specimens.

Equus quagga boebmi: In the single available specimen, the 7th CSP exhibits a robust falcate morphology. The thick attachment of lig. lamina nuchae is the most extensive in the zebrine sample. 
Museum Specimens: The 7th CSP present four morphological profiles - curvate $7 / 27$ (26\%), falcate $11 / 27$ (41\%), scalenate $2 / 27(7 \%)$, and truncate $7 / 27$ (26\%). All forms present robust morphology, even the younger specimens.

\section{DISCUSSION}

This study describes the morphological profile of the 6th and/or 7th CSP in four extant species of Equus and additionally examines museum specimens of pre-domesticated Equus from North America, South America, and Europe. We utilize the methodology of Meyer et al. (2015) to identify specific anatomic structures in order to describe six distinct profiles - cuneate, curvate, falcate, rudimentary, scalenate, and truncate.

In extant species, the 6th CSP exhibits a cuneate profile only in E. asinus, yet this profile is represented in 10 (26\%) C6 museum specimens. This might indicate the specimens, such as E. hermiones and/or E. hydruntinus, are progenitors or close relations of $E$. asinus. Both species are known to have existed in the same geographical regions as pre-domesticated Equus, E. hermiones in North America and E. hydruntinus in Europe (Geigl and Grange 2012; Brown et al. 2015).

Curvate 6th CSP are present in two extant species $-E$. caballus (but only those individuals in which the lig. lamina nuchae attaches to the 2nd to 7th CSPs), and E. przewalskii, in which the lig. lamina nuchae also attaches to the 2nd to 7th CSPs. This morphological profile could therefore be a trait that coincides with the attachment of the lig. lamina nuchae to the 6th CSP and if so, is a likely indicator of a similar ligamentous attachment in museum specimens. A falcate 6th CSP is present only in E. przewalskii and E. quagga boehmi; here the lig. lamina nuchae attachment to the 6th CSP in both species presents a robust morphology A rudimentary 6th CSP is present in only one extant species - E. caballus in which the lig. lamina nuchae attaches to the 2 nd to 5 th CSP. This profile is likely a strong indicator of the absence of lig. lamina nuchae attachments to the 6th CSP, yet no museum specimens presented a rudimentary 6th CSP profile, which further implies these lig. lamina nuchae attachments were present in pre-domesticated Equus. In E. callabus, a 6th CSP with a scalenate profile is associated with both lig. lamina nuchae attachment patterns from the 2 nd to 5 th and 2 nd to 7 th CSP. The latter category presents a profile with a more robust eminence/apex at the site of lig. lamina nuchae attachment. In museum specimens, the scalenate profile occurred in $11 / 39$ (28\%) of the specimens, and although robustness might correlate with the presence of lig. lamina nuchae attachments, it alone is not a reliable indicator, particularly in young animals. Further research into the relationship between the 6th CSP morphology and lig. lamina nuchae attachment is required. Truncate 6th CSP are present only in museum specimens (4/39 or $10 \%$ ); all appeared robust. The cuneate profile is not preserved in any of the fossil 7th CSP samples.

\section{Morphological profiles of the 6th cervical spinous process and four extant species and museum specimens of Equus}

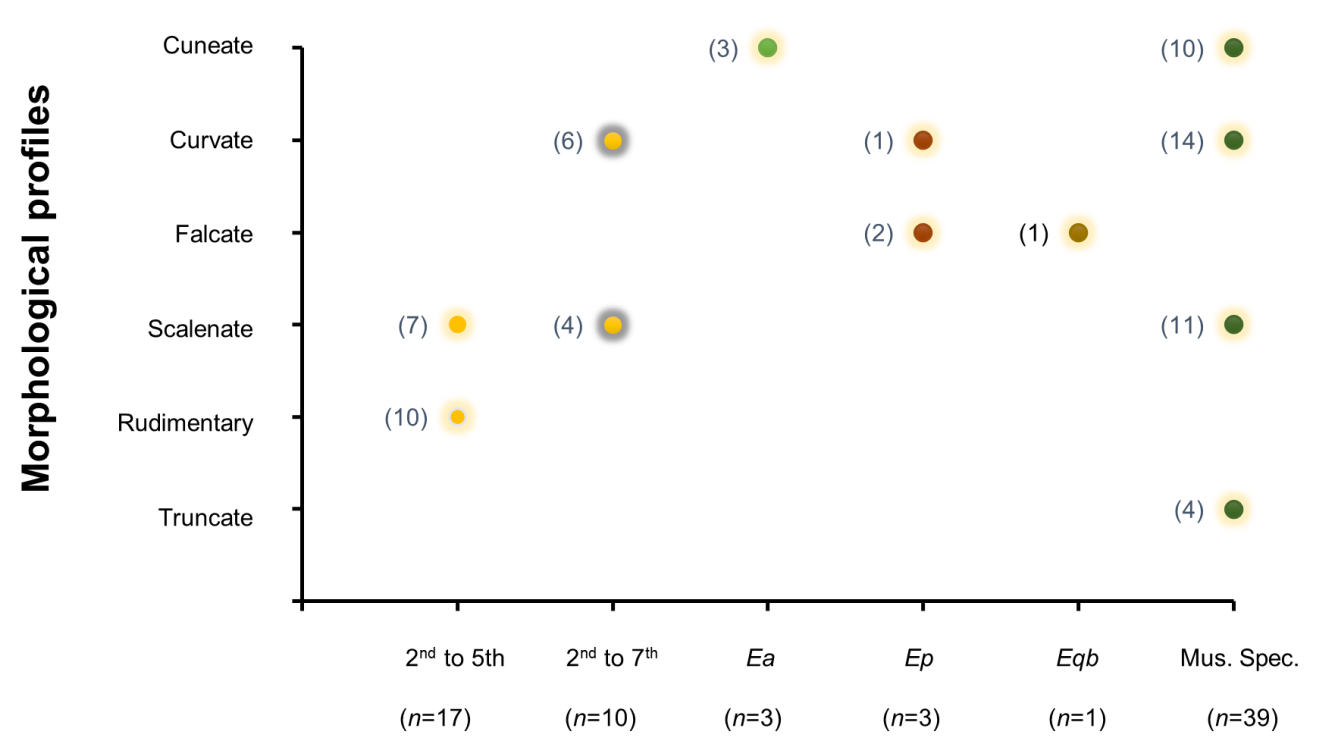

Four extant species and museum specimens of Equus

Figure 4. Morphological profile of the 6th cervical spinous processes in four extant species $(n=33)$ and museum specimens $(n=39)$ of Equus. 
May-Davis et al. - Comparative morphology of spinous processes in Equus

In E. callabus, a curvate 7 th CSP is present in association with both ligament attachment patterns from the 2nd to 5 th CSP (10/17) and 2 nd to 7 th CSP (1/9). This is a strong indication that this profile represents an absence of lig. lamina nuchae attachments. The curvate profile is exhibited by 7 of 27 (26\%) 7th CSP from Rancho La Brea and Tarpits Museum. As a morphological profile, curvate is a strong indicator in extant species that a lig. lamina nuchae attachment to the 7 th CSP is absent, indicating a potentially differing morphology in lig. lamina nuchae attachments in pre-domesticated Equus. However, this sample is geographically isolated and cannot be a definite indicator in all populations. Nevertheless, the robust condition of the 7th CSP of all museum specimens provides an opportunity in future to examine this material for potential enthesis patterns and assess the influence of age on spine morphology. The falcate profile is present in the 7th CSP of all extant species, categories, and museum specimens. However, the two falcate profiles ( $2 *$ in Fig. 5) present in E. callallus (both associated with ligament attaching from the 2 nd to 5th CSP) are low and angled caudally with a straight cranial margin indicative of the scalenate profile. This might be attributed to forces applied from the large attachment of the ligamenta interspinalia along the caudal margin of the 7 th CSP in these two specimens. In addition, these two falcate profiles were comparatively different in orientation to those falcate presentations that were upright and robust in extant specimens with lig. lamina nuchae attachments from the 2 nd to 7 th CSP. A robust falcate profile is exhibited in $11 / 27$ (41\%) of the museum specimens. As evidenced in extant species, this profile is correlated to lig. lamina nuchae attachments the 2 nd to 7 th CSP inclusive, and therefore could been deemed an indicator of lig. lamina nuchae attachments in museum specimens as well.

A scalenate 7th CSP is present only in E. caballus with ligament attaching from the 2 nd to 5 th CSP $(5 / 17)$. The absence of lig. lamina nuchae attachments to the 7 th CSP in extant species suggests that the small percentage $(2 / 27$, or 7\%) of pre-domesticated Equus exhibiting a scalenate 7th CSP also lacked this attachment. Even so, the museum specimens were more robust and the enthesis patterns, if present, would require further research to identify a differing lig. lamina nuchae morphology or other influential factors, such as the maturity of the specimen.

As is the case with the 6th CSP, a truncate 7th CSP is present only in the museum specimens $7 / 27$ (26\%). Since this profile is absent in extant species, the significance of this finding is not yet known; however, the number of specimens present in the museum specimens may be indicative of another species of Equus either known or unknown, or a differing lig. lamina nuchae morphology yet to be identified.

\section{Morphological profiles of the 7th cervical spinous process in four extant species and museum specimens of Equus}

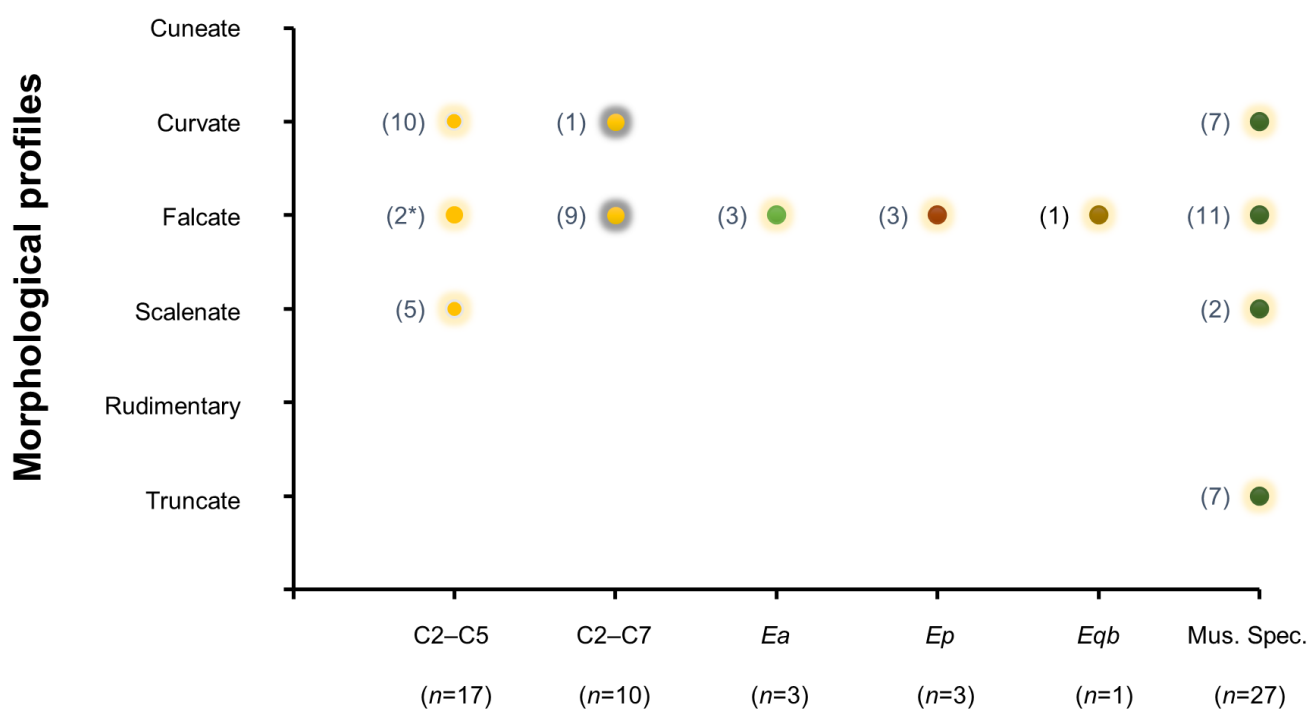

Four extant species and museum specimens of Equus

Figure 5. Morphological profile of the 7 th cervical spinous processes in four extant species $(n=33)$ and museum specimens $(n=27)$ of Equus. *indicates straight cranial margin, apex, and concave caudal margin (representational of falcate and scalenate profiles). 
Specific identification of Equus material held in museum collections has long been a source of consternation for paleontologists; many specimens are labeled simply as 'Equus species?'. This is primarily the result of similar morphology amongst species of Equus and reports of overlapping geographical ranges (Orlando et al 2006). Additionally, complications arise when provenance is lost and disarticulated specimens are retrieved during fishing and dredging techniques, especially in relation to European specimens retrieved from the North Sea (Mol et al. 2006; Lyman 2012).

The long blade-like CSP in early perissodactyls appears to be correlated to a feeding regime that involved browsing. This is still evident in two extant perissodactyl genera (Tapiridae and Rhinoceridae) (MacFadden 1992; Wood et al. 2011; Zsoldos and Licka 2015, Arnold 2020). However, in Equidae, the reduction of CSPs is correlated with a reduction in mechanical constraints on cervical mobility (Denoix 1999). This allows for increased potential for lateroflexion and rotation of the neck in comparison with Hyracotherium, in which dorsoventral flexion predominates (Denoix 1999; Wood et al. 2012). The reduced condition of the CSPs in most extant E. caballus that is correlated with a reduction of the lig. lamina nuchae from the 6th and 7th CSPs is not exhibited in fossil E. caballus, suggesting that this ligament attached to all the relevant CSPs from the 2 nd to 7 th in pre-domestication horses; however, this would require further investigation. Even so, the current study presented morphological findings of the 6th and 7th CSP profiles that might assist paleontologists in specific identification of disarticulated and/or dissociated Equus, as well as inferring the specific morphology of the lig. lamina nuchae in fossil Equus.

\section{ACKNOWLEDGMENTS}

The authors wish to thank Kristen Brabender, Hortabagy National Park, Hungary, for her assistance with Przewalski horses; Wildlands Zoo for their assistance in a Grant's Zebra, Emmen, Netherlands; The Australian College of Equine Podiotherapy for the use of its facilities. Further acknowledgements must be extended to those curators and collection managers of fossil Equus specimens in the following Museums: Dr. Aisling Farrell - Rancho La Brea Tarpits, Los Angeles USA; Professor Lars Werdelin - Swedish Museum of Natural History; Mr. Bram Langeveld - Natuurhistorisch Museum, Rotterdam Netherlands; Dr. Thomas Schossleitner - Berlin Natural History Museum, Germany; and Dr. Elaine Turner - Monrepus Archaeological Research Centre and Museum for Human Behavioural Evolution, Neuwied Germany, and to those authors/editors/publishers of those articles, journals and books cited in this manuscript.

\section{LITERATURE CITED}

Arnold, M.A. Arnold's Glossary of Anatomy. The University of Sydney, Anatomy and Histology. https://anatomy.usyd.edu.au/ glossary/index.cgi Retrieved 12th March 2021.

Arnold, P. 2020. Evolution of the mammalian neck and development, morpho-functional, and paleontological perspectives. Journal of Mammalian Evolution. DOI 10.1007/s10914-02009506-9.

Arnold, P., E. Amson, and M.S. Fischer. 2017. Differential scaling patterns of vertebrae and the evolution of neck length in mammals. Evolution 71:1587-1599.

Brown, K.E., W.A. Akersten, and E. Scott. 2015. Equus occidentalis Leidy from "Asphalto" Kern County, California. Science Series 42:81-99.

Buchholtz, E.A., H.D. Bailin, S.A. Laves, J.T. Yang, M-Y. Chan, and L.E. Drozd. 2012. Fixed cervical count and the origin of the mammalian diaphragm. Evolution and Development 14:399-411.

Denoix, J-M. 1999. Spinal biomechanics and functional anatomy. Veterinary Clinics of North America: Equine Practice 15:27-60.

Dunbar, D.C., J.M. Macpherson, R.W. Simmons, and A. Zarcades. 2008. Stabilization and moonily of the head, neck and trunk in horses during over-ground locomotion; comparisons with humans and other primates. Journal of Experimental Biology 211:3889-3907.

Gasse, H., W. Van Den Broeck, and P. Simeons. 2017. Nomina Anatomica Veterinaria. 6th ed. Published by the Editorial Committee for the World Association of Veterinary Anatomists. Hanover, Ghent, Columbia MO and Rio de Janeiro. 160 pp.

Geigl, E-M. and T. Grange. 2012. Eurasian wild asses in time and space: Morphological versus genetic diversity. Annals of Anatomy 194:88-102.

Gellman, K.S. and J.E.A. Bertram. 2002. The equine nuchal ligament 2: passive dynamic energy exchange in locomotion. Veterinary and Comparative Orthopaedics and Traumatology $1: 7-14$

Getty, R. 1975. Equine osteology; pp. 255-348 in S. Sisson, and J.D. Grossman (eds.), The Anatomy of Domestic Animals. Saunders, Philadelphia USA.

Haussler, K.K. June 2016. Functional anatomy and clinical biomechanics of the equine cervical spine. Paper presented at the AAEP Conference $360^{\circ}$ Pain in the Neck, Fort Collins Colorado.

Lyman, R.L. 2012. A historical sketch on the concepts of archaeological association, context and provenience. Journal of Archaeological Method and Theory 19:207-240.

MacFadden, B.J. 1992. Fossil Horses: Systematics, Paleobiology, and Evolution of the Family Equidae. Cambridge University Press. Cambridge UK. 367 pp.

May-Davis, S. and J. Kleine. 2014. Variations and implications of the gross anatomy in the equine nuchal ligament lamellae. Journal of Equine Veterinary Science 34:1110-1113. 
May-Davis, S., W.Y. Brown, and Z. Vermeulen. 2018. The disappearing lamellae: implications of new findings in the family Equidae suggest the loss of nuchal ligament lamellae on C6 and C7 occurred after domestication. Journal of Equine Veterinary Science 68:108-114.

May-Davis, S., R. Hunter, and W.Y. Brown. 2020a. A comparative morphological study of the ligamentum lamina nuchae and ligamenta interspinalia reveals entheses patterns at the sites of attachment from the second to seventh cervical vertebrae in four extant species of Equus. Vertebrate Anatomy Morphology Palaeontology 8:170-181.

May-Davis, S., F. Minowa, and W.Y. Brown. 2020b. An in situ and ultrasound study of Yonaguni ponies revealed the rare finding of the nuchal ligament lamellae from C2-C7. Journal of Equine Science 31:93-94.

May-Davis, S., Z. Vermeulen, R. Hunter, and W. Brown. 2020c. Rare finding of a full nuchal ligament lamellae with attachment points from C2-C7 in one Australian Stock Horse. Journal of Equine Veterinary Science 84:102847.

Mol, D., K. Post, J.W.F. Reumer, J. van der Plicht, J. de Vos, B. van Geel, G. van Reenen, J.P. Pals, and J. Glimmerveen. 2006. The Eurogeal - first report of the palaeontological, palynological and archaeological investigations of this part of the North Sea. Quaternary International 142-143:178-185.

Muir, J. 1871. The Malayan tapir. Journal of Anatomy and Physiology 6:131-172.

Nussey, D.H., A.J. Wilson, and J.E. Brommer. 2007. The evolutionary ecology of individuals phenotypic platicity in wild populations. Journal of Evolutionary Biology 20:831-844.
Orlando L., M. Mashkour, A. Burke, C.J. Douady, V.

Eisenmann, and C. Hanni. 2006. Geographic distribution of an extinct equid (Equus hydruntinus: Mammalia, Equidae) revealed by morphological and genetical analyses of fossils. Molecular Ecology 15:2083-2093.

Pellow, B.J., M.J. Henwood, and R.C. Carolin. 2010. eFlora: Vascular plants of the Sydney region. The University of Sydney (5th ed). Retrieved 12th March 2021.

Schmaltz, R. 1924. Atlas der Anatomie des Pferdes. Verlagsbuchhandlung von Richard Schoetz: Berlin Germany. Plate $17 b$.

Singh, B. 2018. Dyce, Sack and Wensing's Textbook of Veterinary Anatomy. Elsevier, Missouri, USA. 1606 pp.

Sisson, S. 1910. A Textbook of Veterinary Anatomy. W.B. Saunders, Philadelphia, USA. 724 pp.

Sisson, S. 1953. Equine syndesmology; pp. 209-246 in S. Sisson and J.D. Grossman (eds.), The Anatomy of Domestic Animals. Fourth edition. W.B. Saunders, Philadelphia USA.

Sisson, S. 1975. Equine syndesmology; pp. 349-375 in S. Sisson and J.D. Grossman (eds.), The Anatomy of Domestic Animals. Fifth edition. W.B. Saunders, Philadelphia, USA.

Wood, A.R., R.M. Bebej, C.L. Manz, D.L. Begun, and P.D. Gingerich. 2011. Postcranial functional morphology of Hyracotherium (Equidae, Perissodactyla) and locomotion in the earliest horses. Journal of Mammalian Evolution 18:1-32.

Zsoldos, R.R. and T.F. Licka. 2015. The equine neck and its function during movement and locomotion. Zoology 118:364-376. 